\title{
¿Qué esperan los latinoamericanos de la democracia? Un modelo de análisis de varianza (ANOVA)
}

\author{
Rafael Gustavo Miranda Delgado*
}

\begin{abstract}
RESUMEN
El objetivo de esta investigación es analizar el impacto de las variables materiales en la satisfacción de la democracia en América Latina. La hipótesis que guía esta investigación es que las variables materiales como la seguridad, la reducción de la pobreza, la equidad y el empleo tienen un impacto significativo en la satisfacción de la ciudadanía latinoamericana por sus regímenes democráticos. La investigación demostró que las variables materiales son significativas para entender la satisfacción de la ciudadanía por la democracia. Las variables materiales explican más del 30\% de la satisfacción, o insatisfacción de la democracia en la región. Las variables más demostrativas en la satisfacción de la democracia son la brecha de pobreza, y luego la tasa de victimización.
\end{abstract}

Palabras clave: América Latina, satisfacción por la democracia, variables materiales, democracia, calidad de la democracia.

*What do Latin Americans expect from democracy? An analysis of variance model (ANOVA)

\section{Abstract}

The objective of this research is to analyze the impact of material variables on the satisfaction of democracy in Latin America. The hypothesis that guides this research is that material

* Director cofundador del Grupo de Investigaciones sobre Estudios del Desarrollo y Democracia (GISEDD). Postdoctorado en Ciencias Sociales de la Universidad Central de Venezuela, doctor en Ciencia Política y Relaciones Internacionales de la Universidad Ricardo Palma de Perú, magíster en Relaciones Internacionales de la Universidad Andina Simón Bolívar de Ecuador, economista de la Universidad de Los Andes Venezuela. Actualmente se desempeńa como Profesor de Grado en la Maestría y Doctorado de la Universidad de Los Andes Venezuela. Autor de más de treinta investigaciones sobre desarrollo, democracia y autonomía de América Latina, publicadas en revistas académicas de Latinoamérica, Europa y Asia. Correo electrónico: rafaelgustavomd@hotmail.com

(iD) https://orcid.org/0000-0002-4590-5431 
variables such as security, poverty reduction, equity and employment have a significant impact on the satisfaction of Latin American citizens with their democratic regimes. The research showed that the material variables are significant for understanding citizen satisfaction with democracy. Material variables explain more than $30 \%$ of the satisfaction, or dissatisfaction, of democracy in the region. The most demonstrative variables in the satisfaction of democracy are the poverty gap, and then the victimization rate.

Keywords: Latin America, satisfaction with democracy, material variables, democracy, quality of democracy.

\section{Introducción}

A nivel mundial y en América Latina se observa una insatisfacción por la democracia, y un rechazo por sus principales instituciones como los son los partidos políticos, los parlamentos y el poder legislativo. Cada vez más los ciudadanos están más informados y son más conscientes de sus derechos, por lo que demandan más a sus gobiernos. Estas demandas no solo se circunscriben en a la esfera política entendida en estrecho sentido, sino que también buscan que el gobierno intervenga en otras esferas como la económica. La vertiginosidad de la economía contemporánea hace más vulnerables a la ciudadanía a nivel global, por lo que los temas económicos toman una mayor importancia en el espacio público y en el debate político.

Por ello, el objetivo de esta investigación es analizar el impacto de las variables materiales en la satisfacción de la democracia en América Latina. La hipótesis que guía esta investigación es que las variables materiales como la seguridad, la reducción de la pobreza, la equidad y el empleo tienen un impacto significativo en la satisfacción de la ciudadanía latinoamericana por sus regímenes democráticos.

El artículo se presenta en tres secciones. En democracia y demandas materiales se analizan los vínculos conceptuales y empíricos entre la democracia y su calidad con los resultados en la esfera socioeconómica. En las variables, se reflexiona sobre las variables a utilizar en el modelo ANOVA, satisfacción de la democracia, tasa de victimización, brecha de pobreza, índice Gini y tasa de desempleo, y sobre el comportamiento de los registros y la dirección que toman los valores de las variables entre sí. Finalmente, en modelos ANOVA, se presentan los resultados del análisis econométrico.

\section{Democracia y demandas materiales}

La democracia es un concepto político, hace referencia a la forma de alcanzar y ejercer el poder. No hay un consenso sobre la definición de la democracia, se puede analizar 
desde una perspectiva normativa y desde una formalista. Esta última ha prevalecido en los últimos años.

Por ejemplo, para Bobbio (2009) la democracia es el conjunto de reglas que deben servir para tomar decisiones colectivas, las decisiones que interesan a toda la colectividad, con el máximo de consenso y el mínimo de violencia. Macpherson (1982) define a la democracia como un mecanismo para elegir y autorizar a los gobiernos para que promulguen leyes y adopten decisiones políticas Para Przeworski (2010), hay democracia cuando los que están en el poder pierden las elecciones y los ganadores pasan a ocupar sus cargos. Finalmente, para O'Donnell, (2004) un régimen es democrático cuando el acceso a las principales posiciones de gobierno se logra mediante elecciones competitivas e institucionalizadas, y mantienen diversas libertades políticas.

Pero autores como Dahl (1997) si caracterizan a la democracia de una forma amplia, entendiéndola como el sistema político entre cuyas cualidades se cuenta su disposición a satisfacer entera o casi enteramente a todos sus ciudadanos. Pero para el autor la democracia es solo un ideal, por lo que propone el concepto de poliarquía para referirse a regímenes sustancialmente liberalizados y popularizados.

También hay diferentes formas de valorar la democracia, teóricamente se sugiere generalmente que se haga por el cumplimiento o no de sus procedimientos y cumplimiento de sus mecanismos. Pero la generalidad de la sociedad civil no solo considera estos elementos procedimentales, sino que también se preocupan, tal vez especialmente, por los resultados de la democracia, incluyendo los elementos económicos o materiales. La democracia es un bien en sí mismo, garantiza un conjunto de derechos fundamentales para la justicia y para la libertad, pero adicionalmente también tiene un valor instrumental para alcanzar otros objetivos de estima social, como el desarrollo socioeconómico. La democracia debe buscar satisfacer a la ciudadanía, incluyendo la esfera económica.

Si bien Sartori (1984) sugiere que para hablar de Ciencia Política se deben realizar dos divisiones, primero se debe separar la ciencia de la filosofía y segundo lo político de lo económico y de lo social, nosotros consideramos pertinente un proceso de distinción-conjunción sin desarticulación, y que pueda asociar lo político con su dimensión material.

Si bien lo político y lo económico pueden separase analíticamente, el estado actual de desarticulación no es conveniente para el análisis de estas esferas. Lo político tiende a reducirse a la lucha por el poder, y lo económico a la acción privada de búsqueda de maximización de beneficios en el mercado. Pero si se entienden en forma amplia, a lo político como la construcción del bien común y el gobierno de la esfera pública, y 
a la economía como las relaciones que garantizan al menos las condiciones mínimas materiales de vida y libertad, se hacen evidentes las interrelaciones.

Así pues, las demandas ciudadanas para que el gobierno garantice ciertas condiciones materiales, son coherentes con esta forma de abordar el hecho social. Adicionalmente, se debe advertir, que el desarrollo económico depende del buen Gobierno, y este tiene dos dimensiones fundamentales, la primera referente a la sensibilidad de los gobernantes frente a la demanda de los gobernados, es decir, un buen Gobierno es democrático, y una segunda dimensión referente a la eficiencia de la administración pública, a la burocracia (González y Miranda, 2019). Es decir, no basta con que los gobiernos sean democráticos, adicionalmente se valora la capacidad de su burocracia.

Como señalan Acemoglu y Robinson (2012), las instituciones económicas dan forma a los incentivos económicos, los incentivos para recibir una educación, ahorrar e invertir, innovar y adoptar nuevas tecnologías, pero es el proceso político el que determina cuáles serán las instituciones económicas, y son las instituciones políticas las que determinan cómo funciona este proceso. Las instituciones políticas incluyen constituciones escritas, la calidad de democracia, el poder y la capacidad del Estado para regular y gobernar la sociedad, el reparto de poder y la capacidad de los distintos grupos de actuar colectivamente para conseguir sus objetivos o impedir que otras personas consigan los suyos.

Acemoglu y Robinson (2012) identifican dos tipos de instituciones políticas, las absolutistas y las pluralistas. Si el reparto de poder es restrictivo e ilimitado, las instituciones políticas son absolutistas, quienes ejerzan el poder serán capaces de establecer instituciones económicas para enriquecerse y aumentar su poder a costa de la sociedad, mientras que las instituciones políticas reparten el poder ampliamente en la sociedad y lo limitan, el poder político reside en una amplia coalición o pluralidad de grupos. De estas dos clasificaciones se desprenden las instituciones extractivas y las inclusivas, respectivamente. Las instituciones políticas inclusivas incentivan a establecer instituciones económicas inclusivas, las cuales posibilitan y fomentan la participación de la gran mayoría de la ciudadanía en actividades económicas que aprovechan su talento y habilidades, y que permiten darle las capacidades para gozar de libertades. Por el contrario, las instituciones políticas extractivas generan instituciones económicas extractivas, las cuales incentivan a extraer rentas y riquezas de un subconjunto de la sociedad para beneficiar a un subconjunto distinto.

En América Latina, históricamente se ha observado como la democracia y su calidad, o su ausencia, han sido fundamentales para alcanzar, o impedir, el desarrollo socioeconómico. 
Por ejemplo, Uruguay tuvo una temprana experiencia democrática al ser parte de la denominada primera ola de democratización, es considerada la democracia de mejor calidad de la región, y ha consolidado el más amplio estado de bienestar de América Latina.

Durante los períodos presidenciales de José Batlle y Ordóńez (1903-1907 y 19111915), la actividad política entró en un importante proceso de modernización y la sociedad en un significativo proceso de democratización, y se introdujo una legislación social bastante avanzada para el momento de la región que favoreció especialmente a mujeres, nińos y obreros. Desde las administraciones de Batlle hay una refundación del sistema político en su conjunto, ya que su gestación y posterior desarrollo hizo posible la estructuración de la ideología dominante del Uruguay moderno de un Estado social avanzado para los estándares de América Latina. La democracia uruguaya se desenvolvió no sólo en un espacio formal estrecho, sino que logró ser efectiva y expandirse a la esfera económica y social otorgándole una distinción de calidad y legitimidad que ha perdurar hasta la actualidad (Miranda, 2020a; Miranda, 2019a; Miranda, 2018a).

Adicionalmente, las recientes administraciones del Frente Amplio, Tabaré Vázquez (2005-2010), José Mujica (2010-2015) y de nuevo Tabaré Vázquez (2015-2020) son considerados como los más social democrático de la historia de la región y han logrado combinar la economía de mercado con el Estado de bienestar más desarrollado de América Latina. El desempleo se mantuvo estable y el salario real creció gracias al reforzamiento de la negociación colectiva. Se ampliaron las políticas a favor de las personas en estado de pobreza y de extensión de la ciudadanía social en general con un enfoque de derechos, por canales de tipo burocrático y mediante arreglos institucionales específicos, en un contexto de balance de poderes, negociación y compromisos, lo que permite la veeduría político-administrativa y el gradualismo (Solano y Miranda, 2019; Lanzaro, 2010).

Costa Rica, junto con Uruguay, tiene el Estado de bienestar más amplio de América Latina, es una de las democracias de mejor calidad y la de mayor longevidad de forma interrumpida de la región.

Desde las elecciones presidenciales desde 1953, Costa Rica se ha caracterizado por una democracia estable y de calidad. La resiliencia histórica de la democracia costarricense, en un espacio geopolítico adverso y violento, se debe al denominado consenso social democrático, caracterizado por un gobierno con capacidad para dar respuestas a las demandas socioeconómica de los ciudadanos y por mantener la competencia partidista alrededor del centro político. En la actualidad, con el agotamiento del bipartidismo conformado por el Partido Liberación Nacional (PLN) y el Partido Unidad 
Social Cristiana (PUSC), la democracia costarricense también pudo adaptarse y surgió el Partido Acción Ciudadana (PAC) que retomó la agenda socialdemócrata con las administraciones presidenciales de Luis Guillermo Solís (2014-2018) y de Carlos Alvarado Quesada desde 2018 (Miranda, 2018a; Miranda, 2018b; Miranda, 2017a).

Esto ha permitido que Costa Rica destaque por su significativa tradición en materia de política social. Por ejemplo, desde la administración de Rafael Ángel Calderón Guardia (1940-1944), se implementó una de las reformas sociales más ambiciosas de América Latina para su tiempo, que incluyó la incorporación de garantías sociales en la constitución y códigos laborales, fundando así las bases de la modernidad de un Estado redistribuidor y de la democracia en el país. Luego de la guerra civil (1948), durante el periodo de la Junta Fundadora de la Segunda República, las reformas adelantadas por Calderón se mantuvieron, se creó un sistema de seguridad social universal y se garantizó la educación pública y de calidad. En la actualidad, Costa Rica es uno de los países que realiza mayor inversión social, especialmente destacable es que cuenta con uno de los sistemas de salud más modernos de toda América Latina y posee un sistema de seguridad social integrado por el sector salud y las pensiones que han logrado la mayor cobertura de salud en Centroamérica con un $92 \%$ de la población beneficiada (García y Miranda, 2020; Hangartner y Miranda, 2019a).

Por su parte, en Guatemala el golpe de Estado de 1954 dejó en el poder a Castillo Armas quien masacraría al movimiento social y pondría fin a las reformas sociales iniciadas por el Frente Unido de Partidos y la administración presidencial de Juan José Arévalo (1945-1951). En la contemporaneidad, la democracia guatemalteca sigue siendo frágil y con poca pluralidad de agenda programática, la pobreza sigue siendo extendida y es el país que menos realiza inversión social en América Latina (Miranda, 2018b; Solano y Miranda, 2019; García y Miranda, 2020). Igualmente, Paraguay ha sido dominada por el denominado Partido colorado, el cual ha gobernado de forma hegemónica desde 1947 hasta 1954 y desde 1989 hasta 2008, y ha apoyado a diversas dictaduras, incluyendo a la del general Alfredo Stroessner (1954-1989) que hizo del Paraguay el país con la mayor desigualdad en propiedad de tierras en el mundo. En la actualidad, Paraguay sigue siendo gobernada por el Partido Colorado, cuando Fernando Lugo, un no colorado llegó a la presidencia del país en 2008, varias de sus propuestas de reformas sociales fueron bloqueadas y él fue destituido de manera irregular mediante un juicio político, mientras que el país continúa siendo de los más pobres y desiguales de América Latina (Miranda, 2019b; Miranda, 2020b; Miranda, 2019c).

Finalmente, se debe destacar el caso de venezolano, ya que Venezuela desde 1958 hasta 1978 fue considerada como una de las más estables y mejores democracias de 
América Latina, mientras que en materia socioeconómica se implementó la reforma agraria, se restituyeron los derechos laborales de los trabajadores y empleados, y se implementaron un conjunto de políticas educativas que por sus cualidades y amplitud no habían tenido paralelo en toda la región, lo que generó un alto nivel de satisfacción ciudadana por la democracia. Sin embargo, para la década de 1980 el bipartidismo que sustentaba la democracia venezolana se fue agotando y la satisfaciendo ciudadana por la democracia también fue declinando. En 1998 se eligió como presidente al antisistema y autor de un fallido golpe de Estado al teniente coronel Hugo Chávez, con el cual se fueron deteriorando los elementos democráticos y las condiciones materiales. En la actualidad, con la administración de Nicolás Maduro se ha agudizado el mal gobierno, tanto en su dimensión democrática como en la eficiencia de la burocracia, haciendo de Venezuela uno de los países más pobres de la región (Miranda, 2020c; Miranda, 2020b; Miranda, 2017a; Miranda, 2017b).

Así pues, encontramos significativos vínculos conceptuales y empíricos entre la democracia y su calidad, con el desarrollo socioeconómico y las mejoras materiales en general. Por ello, consideramos que el análisis de la democracia no puede marginar sus derivados económicos. Adicionalmente, como veremos seguido, la ciudadanía también espera que sus gobiernos democráticos se ocupen del bienestar socioeconómico de sus países.

\section{Las variables}

En esta sección, se realizará una breve reflexión sobre las variables a utilizar en el modelo de análisis de varianza (ANOVA) y su relación con la democracia. También se presenta un gráfico de caja realizado con el software $\mathrm{R}$, por cada variable respecto a todos los países en simultáneo, para el periodo de estudio 2002-2016, con el fin de visualizar el comportamiento de los registros y diferenciar la dirección que toman los valores de las variables entre sí.

\subsection{Satisfacción de la democracia}

La satisfacción de la democracia es la variable por explicar en este estudio. Es una variable que expresa la percepción de la ciudadanía. Generalmente, la democracia y su calidad se analizan por sus elementos objetivos y procedimentales, mientras que la satisfacción de la democracia es subjetiva y tiende a centrase en los resultados. Incluso, paradójicamente, la relación entre satisfacción ciudadana y calidad de la democracia no es directa, e incluso puede ser negativa. Por ejemplo, en una democracia en proceso de mejorar su calidad, hay mayor acceso a la información y la ciudadanía es consciente de la corrupción, lo que puede derivar en una baja 
satisfacción por la democracia. Por lo que, siguiendo con el ejemplo, en una democracia de alta calidad que mantiene altos niveles de satisfacción de la ciudadanía, hay un conjunto de mecanismos para evitar la corrupción, y si estos fallan, no solo son expuestos sino también sancionados.

Para la satisfacción de la democracia se utilizó el porcentaje de población de 18 años y más que cree que la democracia es la mejor forma de gobierno. Es una variable que recoge la percepción de los ciudadanos, obtenida de la CEPAL (2021). Seguido en el gráfico 1 se muestra el diagrama de caja para la variable.

Gráfico 1. Diagrama de caja para la variable Satisfacción de la democracia

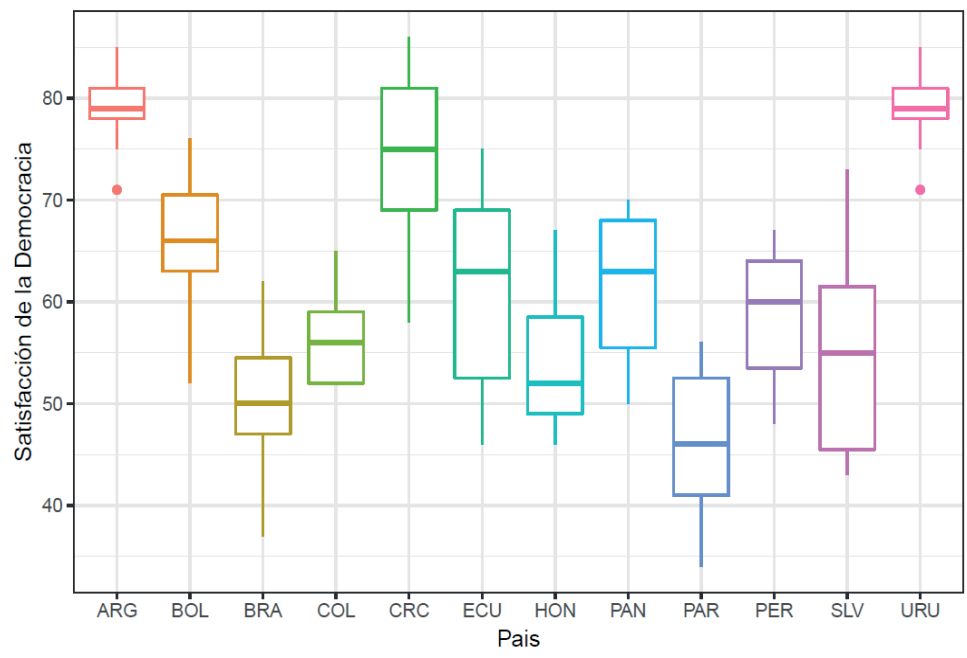

Fuente: CEPAL, 2021.

Para la variable satisfacción de la democracia, se observa que el porcentaje de personas que creen que la democracia es la mejor forma de gobierno varía mucho entre los países de la región. La población de Argentina, Uruguay y Costa Rica tienen los más altos porcentajes durante el periodo de estudio, aunque hay menos variabilidad entre los dos primeros países, mientras que en Costa Rica hay mucha más variabilidad. Por su parte, la población de Brasil y Paraguay no ven la democracia como la mejor forma de gobierno, habiendo más variabilidad en este último país. Finalmente, los habitantes de los demás países de la región se comportan de manera similar, ya que dichas opiniones están en un $60 \%$ aproximadamente, pero se diferencian entre si debido a su variabilidad distinta en sus respectivos registros. 


\subsection{Seguridad}

La garantía de la seguridad física de la ciudadanía no solo es un objetivo primario de la democracia, sino del Estado en general. La violencia generalizada, como la de América Latina, siendo una de las regiones más violentas del mundo, rompe los lazos sociales y genera desconfianza, lesionando así los elementos fundamentales de la cultura democrática. La incapacidad de la democracia de garantizar la seguridad de la ciudadanía tiende a dirigir las preferencias hacia regímenes no democráticos o hacia prácticas no democráticas como darle mayor poder y espacio en la esfera pública a los cuerpos castrenses. Adicionalmente, la violencia impide que las políticas de los gobiernos democráticos lleguen a toda la ciudadanía.

Para la seguridad se utilizó la variable tasa de victimización, la cual se obtiene de la cantidad de población que afirma haber sido víctima de algún delito en los últimos doce meses, como porcentaje de la población total de dieciocho años y más. Es una variable que recoge la percepción de los ciudadanos. Seguido en el gráfico 2 se muestra el diagrama de caja para la variable.

\section{Gráfico 2. Diagrama de caja para la variable Tasa de Victimización}

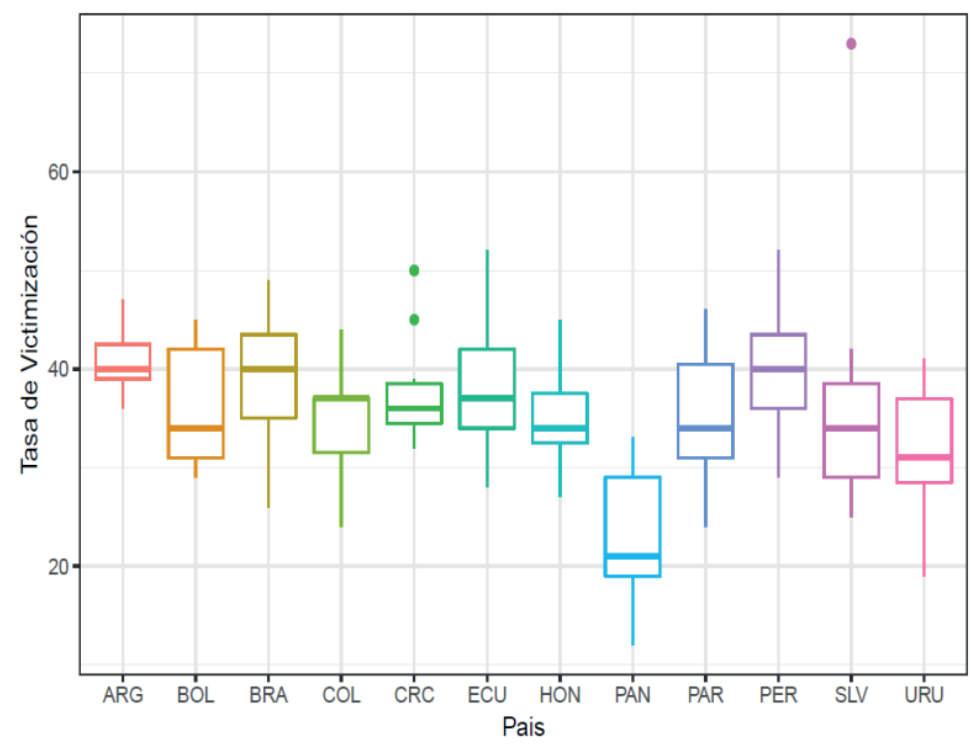

Fuente: CEPAL, 2021.

Para la variable tasa de victimización, se muestra que el porcentaje de personas que no cuentan con una buena seguridad física se comporta de manera similar en todos los países de la región, variando solo en la dispersión de las muestras entre sí. La 
mayoría de los diagramas se encuentran en la misma zona del gráfico, alrededor del $43 \%$. Se debe señalar que se observan tres valores atípicos en el gráfico, los cuales pertenecen uno a El Salvador y los otros dos a Costa Rica.

\subsection{Pobreza}

La pobreza no debe asociarse exclusivamente a la ausencia de dinero, sino a la privación de libertades en general. Sen (2003) advierte que la falta de dinero sufriente es una de las principales causas de la pobreza, pero solo tiene un valor instrumental, mientras que las libertades fundamentales, como saber leer, escribir y contar, tienen un valor constitutivo.

Si bien la pobreza no puede entenderse exclusivamente como la ausencia de dinero, la pobreza monetaria es una buena variable proxy de la pobreza, ya que están intrínsecamente relacionadas, y la primera es causa y consecuencia de la segunda.

La democracia, especialmente la de calidad, espera que la ciudadanía cumpla un significativo y complejo número de funciones. Por ejemplo, debe reconocer y expresar sus preferencias políticas, ejercer su derecho de expresión y para que este sea significativo debe formarse previa y críticamente, asociarse en torno a objetivos comunes, tener capacidad para la rendición de cuenta y la exigencia de resultados, mantener cierto grado de autonomía frente al gobierno. La pobreza dificulta todas estas funciones que se esperan de la ciudadanía.

Por otra parte, al ser la reducción de la pobreza un objetivo tan apremiante, se espera que los regímenes que no logren su reducción tengan un contexto de baja satisfacción de la población, mientras que los logros en esta materia vendrán acompañados de la aprobación ciudadana.

Para la pobreza, se consideró a al porcentaje de la población que vive con menos de 3,2 dólares por día. Seguido en el gráfico 3 se muestra el diagrama de caja para la variable.

En la variable pobreza, se observa que presenta un comportamiento muy distinto dentro de los países de la región, ya que hay países que poseen un mayor porcentaje de personas en estado de pobreza como lo son Bolivia y Honduras. Mientras que Argentina (con un valor atípico), Costa Rica y Uruguay presentan un porcentaje bajo de pobreza. En los demás países del análisis el porcentaje de población en estado de pobreza fluctúa entre un 10 y un $28 \%$. 
Gráfico 3. Diagrama de caja para la variable Pobreza

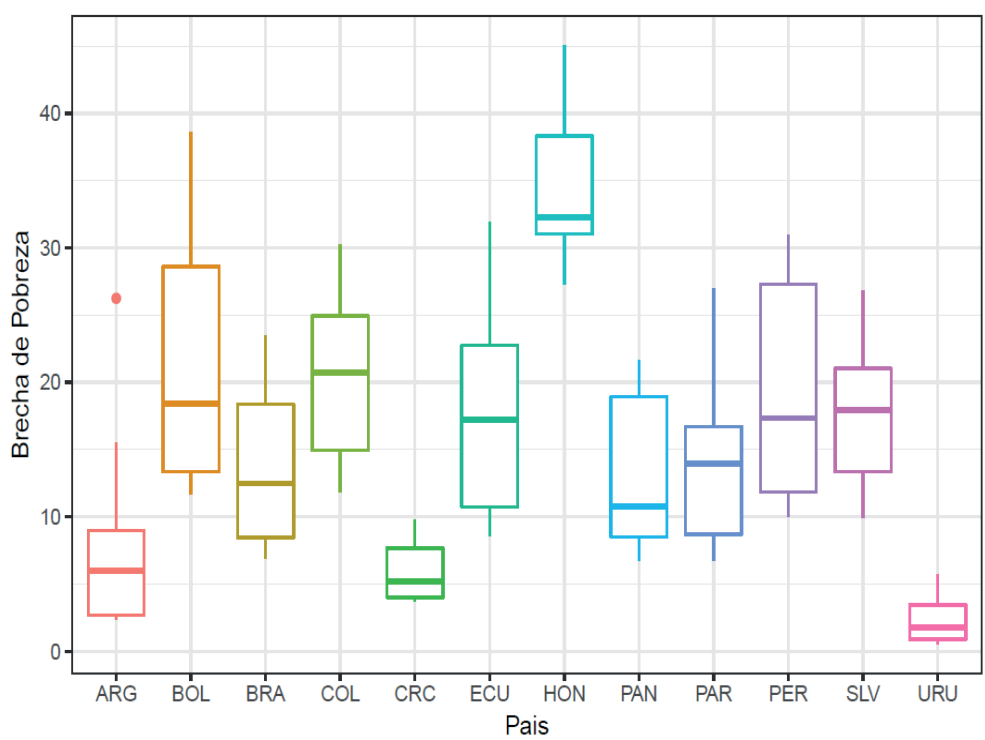

Fuente: CEPAL, 2021.

\subsection{Desigualdad}

La democracia y su calidad están intrínsecamente relacionadas a la igualdad, especialmente a la igualdad política. Por ejemplo, para Morlino (2013), la democracia de calidad es fundamentalmente aquella que permite que sus ciudadanos alcancen igualdad y libertad, mediante el legítimo funcionamiento de sus instituciones.

Si bien, generalmente cuando se habla de igualdad dentro de la teoría de la democracia se hace referencia a la igualdad política, no se puede dejar de lado a la igualdad económica. En una primera instancia, porque la desigualdad económica tiende a generar desigualdad política, como inequidades en el acceso a los mecanismos políticos, dependencias clientelares y diferencias para formar y expresar sus intereses. Adicionalmente, cuando se entiende a la política en su concepto amplio, como construcción de bien común y sus políticas públicas, la reducción de la desigualdad económica es un objetivo político fundamental.

Como señala Bobbio (1986), una de las grandes promesas incumplidas de la democracia es la igualdad, no solo formal sino también sustancia. Con esto, coincide Przeworski (2010), señalando que una de las principales debilidades de la democracia es la incapacidad de generar igualdad en el terreno socioeconómico. 
El elemento fundamental de la igualdad que se relaciona con la democracia es la equidad de oportunidades, es decir, que toda la ciudadanía por derecho tenga acceso igualitario a un conjunto de servicios que les permitan desarrollar sus capacidades. Una sociedad democrática debe impedir las desigualdades que no son producto de diferentes esfuerzos, sino de diferentes oportunidades iniciales derivadas de desigualdades estructurales. Si bien, la equidad no se puede traducir como la reducción de la desigualdad de ingreso, esta es una de las principales fuentes de inequidad de oportunidades.

Para la desigualdad se utilizó el coeficiente de Gini, el cual es un índice que toma valores en el rango $(0,1)$, donde el valor cero corresponde a la equidad absoluta y el uno a la inequidad absoluta. Seguido en el gráfico 4 se muestra el diagrama de caja para la variable.

\section{Gráfico 4. Diagrama de caja para la variable coeficiente de Gini}

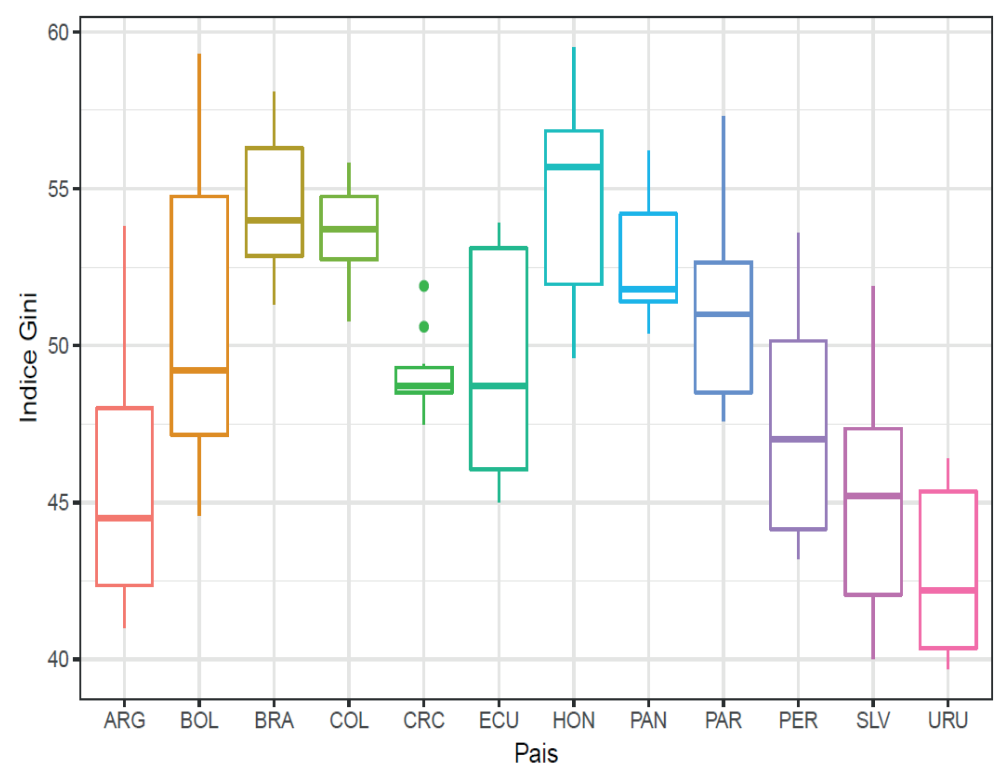

Fuente: Banco Mundial, 2021.

La variable del coeficiente de Gini, evidencia que su comportamiento es muy desigual dentro de la región, ya que existe un grupo de países que poseen un alto coeficiente Gini alrededor del 55\% aproximadamente, y otro grupo de que tienen un índice cercano al $42 \%$. Se debe destacar, que el coeficiente Gini de Costa Rica presenta muy poca variabilidad dentro de las observaciones, sin embargo, tiene dos valores 
anómalos dentro de su gráfico. Y Bolivia tiene la mayor variabilidad dentro de sus registros y no presenta valores atípicos.

\subsection{Desempleo}

El empleo es una de las actividades más significativas para el desarrollo de la personalidad y de la identificación social. Determina la posición social y económica, y el empleo de calidad genera movilidad social y con ella confianza en las instituciones. La remuneración por el empleo es la base de la supervivencia y de la autonomía. Por lo que, el desempleo genera significativo malestar individual y social, tendiendo a lesionar la satisfacción por la democracia.

Para el desempleo, se utilizó la tasa de desempleo, que es el porcentaje de población desocupada con respecto a la población económicamente activa, y se considera población desempleada a las cesantes que son desocupados y que han tenido un trabajo previamente, $\mathrm{y}$ a las que buscan trabajo por primera vez.

Gráfico 5. Diagrama de caja para la variable Tasa de desempleo

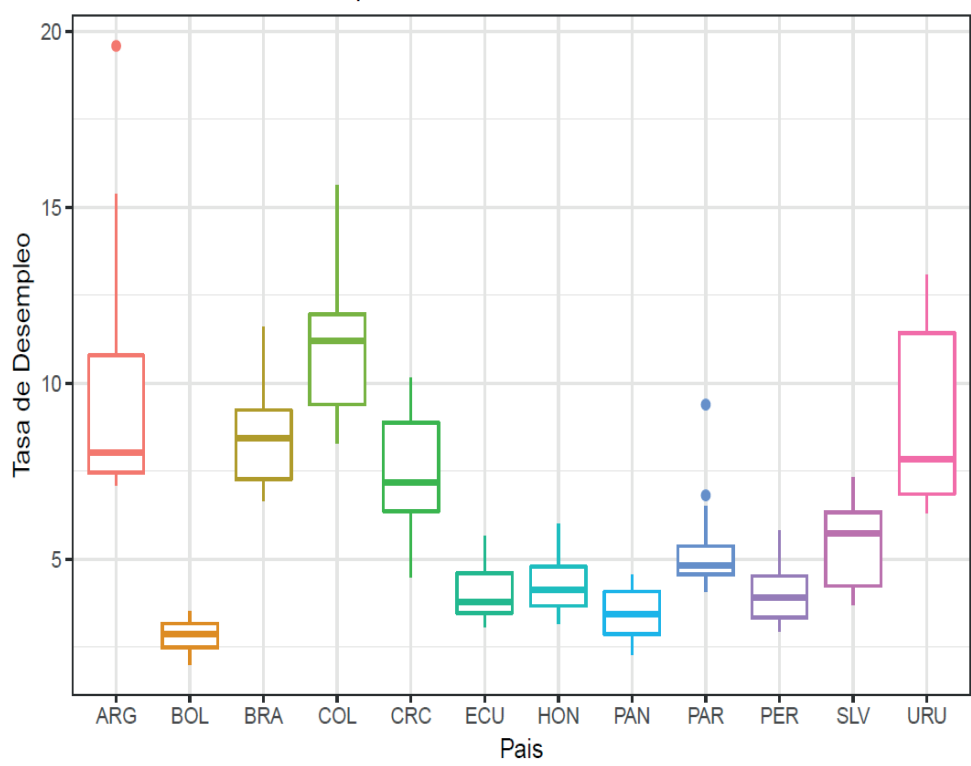

Fuente: Banco Mundial, 2021.

Finalmente, para la variable de la tasa de desempleo, varía mucho dentro de la región, y en general no excede el 15\%. Los países con las tasas más altas de desempleo son Argentina, Brasil, Colombia, Costa Rica y Uruguay; y los de menor desempleo son Bolivia, Ecuador, Honduras, Panamá, Paraguay y Perú. 


\section{Modelos ANOVA}

Los modelos ANOVA son una colección de modelos estadísticos y varios procedimientos asociados entre sí, en el cual la varianza está particionada en ciertos componentes debidos a diferentes variables explicativas. El análisis de la varianza parte de los conceptos de regresión lineal, donde el ANOVA estudia básicamente la influencia de uno o más factores (variables categóricas) sobre una variable de respuesta (variable continua), y tiene como objetivo general establecer si existe diferencia estadística en el efecto promedio de los tratamientos (factores).

Las variables utilizadas en el análisis son satisfacción de la democracia, tasa de victimización, brecha de pobreza, índice Gini y tasa de desempleo. Los datos empleados son extraídos de la Comisión Económica para América Latina y el Caribe (CEPAL) y del Banco Mundial (BM), los cual son observaciones recopiladas en intervalos regulares de forma anual del año 2002 al 2016 para países de Latinoamérica. Por consiguiente, representan un total de 900 muestras aproximadamente con datos faltantes para algunos años en diferentes países.

Debido a la falta de datos disponibles, se depuraron los casos, quedando la matriz de datos integrada solo por los países de Argentina, Bolivia, Brasil, Colombia, Costa Rica, Ecuador, El Salvador, Honduras, Panamá, Paraguay, Perú y Uruguay. Los datos faltantes de esto países se imputaron con la metodología de media.

Se calculó un modelo ANOVA a cada uno de los países, planteándose la variable satisfacción de la democracia como variable dependiente, y tasa de victimización, brecha de pobreza, índice Gini y tasa de desempleo como variables independientes.

Seguido se verifico si los residuos del modelo siguen una distribución normal por medio del test de Shapiro-Wilk, el cual es un test que permite comprobar si los residuos de un modelo siguen una distribución normal.

Finalmente, se calculó el coeficiente de determinación (del modelo para conocer la bondad del ajuste, es decir, cuán bien se ajusta la línea de regresión muestral a los datos.

En la siguiente tabla se muestra el resumen de los resultados y en los anexos las salidas completas del software R. 
Tabla 1. Modelos ANOVA

\begin{tabular}{|c|c|c|c|}
\hline Países & Variable significativa & $\%$ de variabilidad explicada & Normalidad \\
\hline Argentina & Tasa de desempleo & 44,68 & Sí \\
\hline Bolivia & Brecha de pobreza & 33,78 & Sí \\
\hline Brasil & & 20,04 & Sí \\
\hline Colombia & & 26,12 & Sí \\
\hline Costa Rica & $\begin{array}{l}\text { Tasa de victimización } \\
\text { Brecha de pobreza }\end{array}$ & 56,93 & Sí \\
\hline Ecuador & $\begin{array}{l}\text { Tasa de victimización } \\
\text { Brecha de pobreza } \\
\text { Índice de Gini }\end{array}$ & 84,67 & Sí \\
\hline El Salvador & $\begin{array}{l}\text { Tasa de victimización } \\
\text { Brecha de pobreza }\end{array}$ & 56,85 & Sí \\
\hline Honduras & $\begin{array}{l}\text { Tasa de victimización } \\
\text { Tasa de desempleo }\end{array}$ & 58,87 & Sí \\
\hline Panamá & & 20,44 & Sí \\
\hline Paraguay & Brecha de pobreza & 44,13 & Sí \\
\hline Perú & Brecha de pobreza & 42,9 & Sí \\
\hline Uruguay & $\begin{array}{l}\text { Brecha de pobreza } \\
\text { Índice Gini }\end{array}$ & 54,93 & Sí \\
\hline
\end{tabular}

De esta tabla destaca que, la variable más demostrativa en la satisfacción de la democracia es la brecha de pobreza, la cual es significativa en siete modelos distintos, Bolivia, Costa Rica, Ecuador, El Salvador, Paraguay, Perú y Uruguay. La segunda variable más demostrativa fue la tasa de victimización, con cuatro casos, Costa Rica, Ecuador, El Salvador y Honduras. Finalmente, el caso de Ecuador es donde el conjunto de estas variables materiales explica de mejor forma la variabilidad de la satisfacción de la democracia, con un 84,67\%.

En general, estas variables materiales explican más del 30\% de la variabilidad en la satisfacción de la democracia, y en Costa Rica, Ecuador, El Salvador, Honduras y Uruguay explican más del 50\%. Por lo que, efectivamente, las democracias no pueden ignorar sus derivados no políticos. La democracia debe dar respuestas a sus ciudadanos en la esfera política y económica. 
Sin embargo, hay mucha disparidad en la significancia de las variables independientes que se consideraron para medir la opinión de la población en Latinoamérica sobre la satisfacción de la democracia. En los casos de Brasil, Colombia y Panamá ninguna de estas variables materiales fue significativa. Esto pudiese ser porque hay otros elementos materiales o de forma de democracia que son más significativos, como corrupción, representatividad del sistema de partidos políticos, entre otras variables.

\section{Reflexiones finales}

La investigación demostró que las variables materiales son significativas para entender la satisfacción de la ciudadanía por la democracia. Las variables materiales explican más del 30\% de la satisfacción, o insatisfacción de la democracia en la región, y en Costa Rica, Ecuador, El Salvador, Honduras y Uruguay explican más del 50\%. Las variables más demostrativas en la satisfacción de la democracia son la brecha de pobreza, y luego la tasa de victimización.

Conceptual y empíricamente encontramos una fuerte asociación entre democracia y desarrollo socioeconómico, por lo que es coherente que la ciudadanía espere mejoras materiales de sus gobiernos democráticos. La satisfacción ciudadana por la democracia durante el periodo de estudio estuvo alrededor del 60\%, lo que puede considerarse como baja. Esta baja satisfacción de la democracia es explicada en parte por estas variables materiales. La democracia es un bien en sí mismo, pero no puede olvidar sus derivados no políticos.

\section{Referencias bibliográficas}

Acemoglu, D. y Robinson, J. (2012). Por qué fracasan los países. Deusto.

Banco Mundial (2021). Estadisticas de la CEPAL. https://datos.bancomundial.org/

Bobbio, N. (2009). Teoría general de la politica. Editorial Trotta.

Bobbio, N. (1986). El futuro de la democracia. Fondo de Cultura Económica.

CEPAL (2021). Estadísticas de la CEPAL. http://estadisticas.cepal.org/cepalstat/WEB_ CEPALSTAT/Portada.asp

Dahl, R. (1997). La poliarquía. Participación y oposición. Editorial Tecnos, S.A.

García, L. y Miranda, R. (2020). Desarrollo en Centroamérica: hacia una agenda de políticas sociales. Análisis comparado entre el Triángulo Norte y Costa Rica. Cuadernos INTER.C.A.MBIO sobre Centroamérica y el Caribe, 17(2), e41765. https://doi. org/10.15517/c.a.v17i2.41765

Gonzales, C. y Miranda, R. (2019). Desarrollo y buen gobierno. Aplicación al caso venezolano. Teoria y Praxis, 17(35), 21-47. 
Hangartner, A. y Miranda, R. (2019). Estado del bienestar y justicia distributiva en América Latina. Un análisis crítico. Revista ABRA, 39(58), 9-29. https://doi.org/10.15359/ abra.39-58.1

Lanzaro, J. (2010). Uruguay: un gobierno social democrático en América Latina. Revista Uruguaya de Ciencia Politica, 19(1), 45-68.

Macpherson, C. (1982). La democracia liberal y su época. Alianza Editorial, S.A.

Miranda, R. (2020a). Sistemas políticos del Cono Sur y Brasil. Análisis Histórico Comparado desde la independencia hasta la actualidad. ACADEMO. Revista de Investigación en Ciencias Sociales y Humanidades, 7(1), 67-81.

Miranda, R. (2020b). Pobreza, desigualdad y vulnerabilidad en América Latina (20002020). Americanía. Revista de Estudios Latinoamericanos, (11), 56-90. https://doi. org/10.46661/americania.5200

Miranda, R. (2020c). Del populismo al autoritarismo legalizado. Análisis Histórico Comparado entre Venezuela y Nicaragua. Revista de Ciencias Sociales y Humanidades, 29(57), 23-38. http://doi.org/10.20983/noesis.2020.1.2

Miranda, R. (2019a). Sistemas políticos y quiebre del bipartidismo. Análisis Histórico Comparado entre Uruguay y Venezuela. Contextualizaciones Latinoamericanas, 11(20), 1-9. https://doi.org/10.30545/academo.2020.ene-jun.7

Miranda, R. (2019b). Instituciones Políticas y Desigualdad de Ingresos en América Latina: Desde la Independencia hasta la Actualidad. Asian Journal of Latin American Studies, 32(2), 1-24.

Miranda, R. (2019c). Sistemas políticos con partidos hegemónicos. Análisis Histórico Comparado entre México y Paraguay. Antrópica. Revista de Ciencias Sociales y Humanidades, 5(10), 117-136.

Miranda, R. (2018a). Democracia de alta calidad en América Latina. Análisis histórico comparado entre Chile, Costa Rica y Uruguay. Política. Revista de Ciencia Politica, 56(1), 89-106.

Miranda, R. (2018b). Political Systems in Central America. A Compared Historical Analysis. Temas de nuestra América, 34(64), 83-106.

Miranda, R. (2017a). ¿Qué ha sido de las democracias de la segunda ola? Análisis Histórico Comparado entre Costa Rica, Colombia y Venezuela. Ciencia Política, 12(24), 281-304.

Miranda, R. (2017b). Industrialización y desindustrialización en Venezuela. Un análisis histórico. Ensayos de economía, 27(50), 87-101. https://doi.org/10.15446/ede. v27n50.66523

Morlino, L. (2013). La calidad de las democracias en América Latina. IDEA Internacional.

O’Donnell, G. (2004). Acerca del estado en América Latina Contemporánea: diez tesis para su discusión. En PNUD. (Ed.), La democracia en América Latina hacia una democracia de ciudadanas y ciudadanos: contribuciones para el debate (pp. 149-191). PNUD.

Przeworski, A. (2010) ¿Qué esperar de la democracia?: límites y posibilidades del autogobierno. Siglo XXI. 
Sartori, G. (1984). La política. Lógica y método en las ciencias sociales. Fondo de Cultura Económica.

Sen, A. (2003). Desarrollo y libertad. Editorial Planeta.

Solano, E. y Miranda, R. (2019). Estado de bienestar en América Latina: hacia una tipología política. Análisis comparado entre Uruguay, México y Guatemala. Cuadernos del CLAEH, 38(110), 253-272.

Fecha de recepción: 9 de marzo de 2021 Fecha de aprobación: 11 de mayo de 2021 


\section{Anexos}

\section{Salidas de ANOVA}

Modelo ANOVA para Argentina

$\begin{array}{lrrrrr} & \text { Df } & \text { Sum Sq } & \text { Mean Sq } & \text { F value } & \operatorname{Pr}(>F) \\ \text { Tasa_Vic_Arg } & 1 & 3.11 & 3.11 & 0.279 & 0.6090 \\ \text { Brech_Pobreza_Arg } & 1 & 11.65 & 11.65 & 1.042 & 0.3313 \\ \text { Ind_Gini_Arg } & 1 & 2.43 & 2.43 & 0.218 & 0.6508 \\ \text { Tasa_Desempleo_Arg } & 1 & 73.06 & 73.06 & 6.539 & 0.0285 * \\ \text { Residuals } & 10 & 111.74 & 11.17 & & \end{array}$

Fuente: Salida de R. Elaboración propia.

Modelo ANOVA para Bolivia

$\begin{array}{lrrrrr} & \text { Df } & \text { Sum Sq } & \text { Mean Sq F value } \operatorname{Pr}(>\mathrm{F}) \\ \text { Tasa_Vic_Bol } & 1 & 0.1 & 0.07 & 0.001 & 0.9705 \\ \text { Brech_Pobreza_Bol } & 1 & 240.4 & 240.35 & 4.795 & 0.0534 \\ \text { Ind_Gini_Bol } & 1 & 0.6 & 0.64 & 0.013 & 0.9124 \\ \text { Tasa_Desempleo_Bol } & 1 & 14.6 & 14.61 & 0.291 & 0.6011 \\ \text { Residuals } & 10 & 501.3 & 50.13 & & \end{array}$

Fuente: Salida de R. Elaboración propia.

Modelo ANOVA para Brasil

$\begin{array}{lrrrrr} & \text { Df } & \text { Sum Sq } & \text { Mean Sq } & \text { F value } & \text { Pr }(>F) \\ \text { Tasa_Vic_Bra } & 1 & 2.9 & 2.87 & 0.039 & 0.848 \\ \text { Brech_Pobreza_Bra } & 1 & 69.3 & 69.35 & 0.938 & 0.356 \\ \text { Ind_Gini_Bra } & 1 & 9.5 & 9.45 & 0.128 & 0.728 \\ \text { Tasa_Desempleo_Bra } & 1 & 103.7 & 103.66 & 1.402 & 0.264 \\ \text { Residuals } & 10 & 739.6 & 73.96 & & \end{array}$

Fuente: Salida de R. Elaboración propia. 
Modelo ANOVA para Colombia

$\begin{array}{lrrrrr} & \text { Df } & \text { Sum Sq } & \text { Mean Sq } & \text { F value } & \text { Pr }(>F) \\ \text { Tasa_Vic_Col } & 1 & 5.47 & 5.47 & 0.296 & 0.599 \\ \text { Brech_Pobreza_Col } & 1 & 57.88 & 57.88 & 3.128 & 0.107 \\ \text { Ind_Gini_Col } & 1 & 1.10 & 1.10 & 0.059 & 0.813 \\ \text { Tasa_Desempleo_Col } & 1 & 0.96 & 0.96 & 0.052 & 0.824 \\ \text { Residuals } & 10 & 185.00 & 18.50 & & \end{array}$

Fuente: Salida de R. Elaboración propia.

Modelo ANOVA para Costa Rica

$\begin{array}{lrrrrr} & \text { Df } & \text { Sum Sq } & \text { Mean Sq F value } & \text { Pr }(>F) \\ \text { Tasa_Vic_CostR } & 1 & 239.0 & 239.00 & 5.695 & 0.0382 * \\ \text { Brech_Pobreza_CostR } & 1 & 258.1 & 258.14 & 6.151 & 0.0325 * \\ \text { Ind_Gini_CostR } & 1 & 6.4 & 6.43 & 0.153 & 0.7037 \\ \text { Tasa_Desempleo_CostR } & 1 & 51.1 & 51.15 & 1.219 & 0.2955 \\ \text { Residuals } & 10 & 419.7 & 41.97 & & \end{array}$

Fuente: Salida de R. Elaboración propia.

Modelo ANOVA para Ecuador

$\begin{array}{lrrrrrl} & \text { Df } & \text { Sum Sq } & \text { Mean Sq } & \text { F value } & \text { Pr }(>F) & \\ \text { Tasa_Vic_Ecu } & 1 & 106.0 & 106.0 & 5.558 & 0.0401 * \\ \text { Brech_Pobreza_Ecu } & 1 & 825.9 & 825.9 & 43.299 & 6.23 e-05 & * * * \\ \text { Ind_Gini_Ecu } & 1 & 80.9 & 80.9 & 4.240 & 0.0665 & . \\ \text { Tasa_Desempleo_Ecu } & 1 & 40.4 & 40.4 & 2.120 & 0.1761 \\ \text { Residuals } & 10 & 190.7 & 19.1 & & & \end{array}$

Fuente: Salida de R. Elaboración propia.

Modelo ANOVA para El Salvador

\begin{tabular}{|lrcrll|}
\hline & Df & Sum Sq Mean Sq F value Pr(>F) \\
Tasa_Vic_Sal & 1 & 315.3 & 315.3 & 5.398 0.0425 * \\
Brech_Pobreza_Sal & 1 & 329.4 & 329.4 & 5.640 & $0.0390 *$ \\
Ind_Gini_Sal & 1 & 24.0 & 24.0 & 0.411 & 0.5360 \\
Tasa_Desempleo_Sal & 1 & 100.9 & 100.9 & 1.727 & 0.2181 \\
Residuals & 10 & 584.1 & 58.4 & & \\
\hline
\end{tabular}

Fuente: Salida de R. Elaboración propia. 
Modelo ANOVA para Honduras

$\begin{array}{lrrrrrr} & \text { Df } & \text { Sum Sq } & \text { Mean Sq } & \text { F value } & \text { Pr }(>F) \\ \text { Tasa_Vic_Hon } & 1 & 215.97 & 215.97 & 8.966 & 0.0135\end{array}$ *

Fuente: Salida de R. Elaboración propia.

Modelo ANOVA para Panamá

$\begin{array}{lrrrrr} & \text { Df } & \text { Sum Sq } & \text { Mean Sq } & \text { F value } & \text { Pr }(>F) \\ \text { Tasa_Vic_Pan } & 1 & 121.6 & 121.62 & 2.030 & 0.185 \\ \text { Brech_Pobreza_Pan } & 1 & 29.6 & 29.60 & 0.494 & 0.498 \\ \text { Ind_Gini_Pan } & 1 & 2.3 & 2.34 & 0.039 & 0.847 \\ \text { Tasa_Desempleo_Pan } & 1 & 0.3 & 0.33 & 0.005 & 0.942 \\ \text { Residuals } & 10 & 599.0 & 59.90 & & \end{array}$

Fuente: Salida de R. Elaboración propia.

Modelo ANOVA para Paraguay

$\begin{array}{lrrrrr} & \text { Df } & \text { Sum Sq } & \text { Mean Sq F value Pr }(>F) \\ \text { Tasa_Vic_Par } & 1 & 85.0 & 84.96 & 2.119 & 0.1761 \\ \text { Brech_Pobreza_Par } & 1 & 208.1 & 208.14 & 5.191 & 0.0459 \\ \text { Ind_Gini_Par } & 1 & 2.0 & 2.04 & 0.051 & 0.8261 \\ \text { Tasa_Desempleo_Par } & 1 & 21.6 & 21.62 & 0.539 & 0.4797 \\ \text { Residuals } & 10 & 401.0 & 40.10 & & \end{array}$

Fuente: Salida de R. Elaboración propia.

Modelo ANOVA para Perú

$\begin{array}{lrrrrr} & \text { Df } & \text { Sum Sq } & \text { Mean Sq } & \text { F value } & \text { Pr }(>F) \\ \text { Tasa_Vic_Per } & 1 & 0.0 & 0.01 & 0.000 & 0.9878 \\ \text { Brech_Pobreza_Per } & 1 & 201.0 & 201.04 & 6.321 & 0.0307 \\ \text { Ind_Gini_Per } & 1 & 36.1 & 36.12 & 1.136 & 0.3116 \\ \text { Tasa_Desempleo_Per } & 1 & 1.7 & 1.73 & 0.054 & 0.8202 \\ \text { Residuals } & 10 & 318.0 & 31.80 & & \end{array}$

Fuente: Salida de R. Elaboración propia. 
Modelo ANOVA para Uruguay

$\begin{array}{lrrrrr} & \text { Df } & \text { Sum Sq } & \text { Mean Sq } & F \text { value } & \text { Pr }(>F) \\ \text { Tasa_Vic_Uru } & 1 & 23.93 & 23.93 & 2.509 & 0.1443 \\ \text { Brech_Pobreza_Uru } & 1 & 47.78 & 47.78 & 5.010 & 0.0491 \\ \text { Ind_Gini_Uru } & 1 & 37.37 & 37.37 & 3.918 & 0.0759 \\ \text { Tasa_Desempleo_Uru } & 1 & 7.16 & 7.16 & 0.750 & 0.4066 \\ \text { Residuals } & 10 & 95.37 & 9.54 & & \end{array}$.

Fuente: Salida de R. Elaboración propia. 